\title{
Erradicación del analfabetismo funcional en el noreste de Brasil
}

\section{Functional illiteracy eradication in Brazilian Northeast}

DOI: $10.46932 /$ sfjdv2n4-011

Received in: Jun 1st, 2021

Accepted in: Sep 30th, 2021

\author{
Leonor Scliar-Cabral \\ Universidade Federal de Santa Catarina, Brasil \\ E-mail: leonorsdc20@gmail.com
}

\section{RESUMEN}

OBJETIVOS: Mostrar las bases del Sistema de Alfabetización Scliar, creado para erradicar el analfabetismo funcional en Brasil, aplicado en dos municipios del noreste de Brasil.

METODOLOGÍA: Investigación intervencionista que consistió en la educación continua en línea semanal de educadores involucrados en la enseñanza de la alfabetización a niños de $1^{\circ}$ y $2^{\circ}$ grado en escuelas públicas de São José da Laje, AL y Lagarto, SE, con metodología innovadora y material pedagógico, basados en los avances de la neurociencia, la lingüística, psicolingüística y neuropsicologia. Los instrumentos de evaluación de resultados fueron la Evaluación Nacional de Alfabetización (ANA), el Más Alfabetización y la prueba de Fluidez, todos del Ministerio de Educación de Brasil.

RESULTADOS: Los niños de São José da Laje dieron un salto gigante en la competencia lectora, considerando el nivel más alto y deseable alcanzado por solo el $1.39 \%$ de los estudiantes de 8 años en el tercer año, según la ANA de 2016: después de la adopción del SSA, el municipio saltó al $71 \%$ de los estudiantes, a la edad de 7 años, en el mismo nivel, no en el $3^{\circ}$, sino en el $2^{\circ}$ año, según el Más Alfabetización, em 2018. La ANA constato en el nivel más bajo 1, en el estado de Sergipe un porcentaje muy alto de 45,28\% y en el 4, el nivel de competencia deseable, solo el 3.02. En la comparación de tales resultados, con los obtenidos en el Más Alfabetización, los dos colegios de Lagarto, también en el estado de Sergipe, que han adoptado la SSA desde 2017, constatase que en el nivel 1 hubo un porcentaje de solo 8,7 y 9,1, mientras en el nivel deseado, 3, el más alto las dos escuelas alcanzaron el 34,8 y el 31,8\%.

CONCLUSIONES: Los resultados muestran que es posible erradicar el analfabetismo funcional, si se produce un cambio en las políticas públicas de alfabetización, a favor de la formación continua de alfabetizadores, de metodologías y material pedagógico, basados en las ciencias que abordan el lenguaje verbal.

Palabras clave: Analfabetismo funcional, Sistema Scliar de alfabetización, Noreste de Brasil, Reciclaje neuronal, Conciencia fonémica.

\begin{abstract}
GOALS: Showing Scliar Literacy System fundamentals, created to eradicate functional illiteracy in Brazil, applied in two cities in the Brazilian northeast.

METHODOLOGY: Interventional research that consisted of weekly online educators' continuing education involved in teaching literacy to $1^{\text {st }}$ and $2^{\text {nd }}$ grade children in São José da Laje, AL and Lagarto, SE public schools, with innovative methodology and pedagogical material, based on advances in neuroscience, linguistics, psycholinguistics and neuropsychology. The results evaluation instruments were the National Early Literacy Assessment (ANA), the More Early Literacy and the Fluency test, all from the Brazilian Ministry of Education.

RESULTS: São José da Laje children made a giant leap in reading proficiency, considering the highest and most desirable level reached by only $1.39 \%$ of 8 -year-old students in the third year, according to the
\end{abstract}


2016 ANA: after the SSA adoption, the municipality jumped to $71 \%$ of the students, at the age of 7 , at the same level, not in the $3^{\text {rd }}$, but in the $2^{\text {nd }}$ year, according to the More Early Literacy, in 2018. The ANA found in the lowest level 1, in Sergipe state a very high percentage of $45.28 \%$ and in 4 , the desirable level of competence, only $3.02 \%$. Comparing these results, with those obtained by More Early Literacy, the Lagarto two schools, also in the state of Sergipe, which have adopted the SSA since 2017, it was found that in level 1 there was a percentage of only 8.7 and 9.1, while at the desired level, 3, the highest, the two schools reached 34.8 and $31.8 \%$.

CONCLUSIONS: The results show that it is possible to eradicate functional illiteracy, if there is a change in public literacy policies, favoring continuous training of early literacy teachers, methodologies and pedagogical material, based on the sciences that address verbal language.

Keywords: Functional illiteracy, Scliar Early Literacy System, Brazilian Northeast, Neural recycling, Phonemic awareness.

\section{INTRODUCCIÓN}

Los últimos resultados publicados por Inaf (2018), el Instituto Nacional de Alfabetización Funcional, la institución más reputada y rigurosa en encuestas de hogares en el área, en Brasil, revelan que hubo una leve mejoría en relación a los datos de 2001-2002, pero el situación aún es muy grave, ya que el $8 \%$ de la población sigue siendo totalmente analfabeta y solo el 12\% es competente, es decir, capaz de una lectura crítica de los textos que circulan socialmente, insertos en la sociedad de la información y capaces de ingresar a los más calificados puestos del mercado laboral.

Tabla 1 - Niveles de alfabetismo en Brasil según el Inaf (2001-2018)

\begin{tabular}{|l|l|l|l|l|l|l|l|l|l|}
\hline Nível & $\begin{array}{l}\mathbf{2 0 0 1} \\
\mathbf{2 0 0 2}\end{array}$ & $\begin{array}{l}\mathbf{2 0 0 2} \\
\mathbf{2 0 0 3}\end{array}$ & $\begin{array}{l}\mathbf{2 0 0 3} \\
\mathbf{2 0 0 4}\end{array}$ & $\begin{array}{l}\mathbf{2 0 0 4} \\
\mathbf{2 0 0 5}\end{array}$ & $\mathbf{2 0 0 7}$ & $\mathbf{2 0 0 9}$ & $\mathbf{2 0 1 1}$ & $\mathbf{2 0 1 5}$ & $\mathbf{2 0 1 8}$ \\
\hline BASE & $\mathbf{2 0 0 0}$ & $\mathbf{2 0 0 0}$ & $\mathbf{2 0 0 1}$ & $\mathbf{2 0 0 2}$ & $\mathbf{2 0 0 2}$ & $\mathbf{2 0 0 2}$ & $\mathbf{2 0 0 2}$ & $\mathbf{2 0 0 2}$ & $\mathbf{2 0 0 2}$ \\
\hline Analfabeto & $12 \%$ & $13 \%$ & $12 \%$ & $11 \%$ & $9 \%$ & $7 \%$ & $6 \%$ & $4 \%$ & $8 \%$ \\
\hline Rudimentar & $27 \%$ & $26 \%$ & $26 \%$ & $26 \%$ & $25 \%$ & $20 \%$ & $21 \%$ & $23 \%$ & $22 \%$ \\
\hline Elementar & $28 \%$ & $29 \%$ & $30 \%$ & $31 \%$ & $32 \%$ & $35 \%$ & $37 \%$ & $42 \%$ & $34 \%$ \\
\hline Intermediario & $20 \%$ & $21 \%$ & $21 \%$ & $21 \%$ & $21 \%$ & $27 \%$ & $25 \%$ & $23 \%$ & $25 \%$ \\
\hline Proficiente & $12 \%$ & $12 \%$ & $12 \%$ & $12 \%$ & $13 \%$ & $11 \%$ & $11 \%$ & $8 \%$ & $12 \%$ \\
\hline Total2 & $100 \%$ & $100 \%$ & $100 \%$ & $100 \%$ & $100 \%$ & $100 \%$ & $100 \%$ & $100 \%$ & $100 \%$ \\
\hline Analfabeto Funcional* & $39 \%$ & $39 \%$ & $37 \%$ & $37 \%$ & $34 \%$ & $27 \%$ & $27 \%$ & $27 \%$ & $29 \%$ \\
\hline $\begin{array}{l}\text { Funcionalmente } \\
\text { Alfabetizados* }\end{array}$ & $61 \%$ & $61 \%$ & $63 \%$ & $63 \%$ & $66 \%$ & $73 \%$ & $73 \%$ & $73 \%$ & $71 \%$ \\
\hline
\end{tabular}

Fuente: Inaf 2001-2018, p. 8.

Fue un panorama tan desolador que me llevó, en 2007, a comprometerme con la erradicación del analfabetismo funcional: llegué a la conclusión de que el mejor aporte sería garantizar una alfabetización de calidad, ya que es en los primeros años que se decide el destino del estudiante, si será o no un lector competente, en completo ejercicio de la ciudadanía y, para alcanzar el objetivo, es necesario formar educadores, basando la metodología y el material didáctico en los avances de las ciencias que abordan la estructura, funcionamiento, adquisición y aprendizaje del lenguaje verbal: la neurociencia, la lingüística, 
la psicolingüística, la sociolingüística y neuropsicologia. Fue en este contexto que creé el Sistema de Alfabetización Scliar (SSA), que voy a describir.

Una propuesta que indica la importancia de implementar nuevas metodologías educativas y la mentalidad de los maestros se encuentra en Rodríguez (2021, p. 5854).

La gran motivación para desarrollar el Sistema Scliar de Alfabetización, para todos aquellos que se involucran en el proceso de alfabetización con el fin de formar un lector competente, se deriva del hecho de que, en primer lugar, a pesar del esfuerzo de los educadores, la tasa de analfabetismo funcional es todavía muy alto.

Los profesores son conscientes de ello y más ansiosos que nadie de que sus alumnos aprendan a leer para comprender los textos que circulan a su alrededor, ya sean periódicos, libros, poemas, avisos, instrucciones o información en el ordenador; y también para escribir para que se hagan entender cuando tienen que enviar una carta o hacer un examen para conseguir un trabajo, o para entrar a la universidad.

El objetivo es hacer que los alfabetizadores:

1. obtengan mejores resultados con sus alumnos, para que se sientan más seguros, desarrollando el gusto por la lectura y la escritura;

2. comprendan mejor las dificultades de sus alumnos y sepan cómo superarlas;

3. Dispongan de material de calidad, basado en las últimas investigaciones en neurociencia, lingüística, psicolingüística y neuropsicología de la lectura, sabiendo para qué sirve cada ejercicio y cómo debe aplicarse.

Los fundamentos de la propuesta se basan en la teoría y práctica más avanzadas de las ciencias que se ocupan del lenguaje verbal y son:

- La adquisición del sistema oral se produce de forma natural y espontánea en niños que no tienen grandes trastornos sensitivomotores, perceptivos o cognitivos: las primeras palabras se producen alrededor del primer año de edad; el sistema escrito se construye en el contexto de la enseñanzaaprendizaje de manera sistemática e intensiva, cuando el niño ya ha alcanzado una cierta madurez cognitiva, lingüística y emocional.

Si bien la comunicación oral sea una cuestión de supervivencia humana, desde que empezó el proceso de humanización, la escritura, entendida como un modo secundario, distinto de la pintura, del dibujo u de otros medios de registro cultural, apareció muy recientemente (aproximadamente 5.000 años antes de Cristo).

Aplicación: En la mayoría de los casos, el niño necesita venir a la escuela principalmente para aprender a leer y escribir, incluidos los símbolos matemáticos. 
- En todo aprendizaje, para saber producir hay que saber comprender, es decir, antes de hablar, el niño debe comprender lo que le dicen los adultos y así empezar a dominar el idioma, para que luego pueda emitir sus primeras palabras. Lo mismo ocurre con el lenguaje escrito: sin saber leer, el niño no podrá entender ni siquiera lo que "escribió".

Aplicación: la alfabetización no puede empezar por la enseñanza de la escritura de forma aislada. Incluso puede iniciarla simultáneamente con la escritura, siempre que el niño haya aprendido a reconocer las diferencias entre las letras y los valores del grafema en la lectura.

- Uno de los grandes descubrimientos de la neurociencia de la lectura es que las neuronas que procesan las imágenes visuales están programadas para simetrizar la información: "Las neuronas visuales fueron programadas biológicamente para simetrizar la información. Cada vez que un hemisferio aprende nueva información visual, este rasgo de memoria se transmite inmediatamente al otro hemisferio " (Dehaene, 2012, p. 292).

Para que las neuronas reconozcan cualquier cosa como la misma, se descuida la diferencia entre izquierda y derecha, lo que se llama simetrización: si el asa de una taza está a la derecha o a la izquierda, reconoceremos la taza como la misma.

Sin embargo, para el reconocimiento de letras, es decir, de las diferencias que se presentan entre ellas, es necesario reciclar las neuronas para que aprendan a distinguir la dirección de los rasgos de las letras: habrá que rehacer la percepción durante la alfabetización, porque colocar las tres pequeñas líneas horizontales paralelas solo puede estar a la derecha de la línea vertical para formar la letra E. Aún más difícil es reconocer la diferencia entre las letras d / b o entre q / p, la cual radica solo en el hecho de que las primeras letras de cada par estén con el semicírculo a la izquierda del rasgo vertical y las segundas, a la inversa, con el semicírculo a la derecha del rasgo vertical (espejo horizontal).

Esto requiere un trabajo de enseñanza minucioso y continuo. Por ello, desarrollo la propuesta de Montessori (1997) de activar al máximo las distintas entradas sensoriales para ganar la batalla de disimetrizar las características que diferencian las letras y los símbolos matemáticos entre sí, reciclando las neuronas lectoras ubicadas en el área occipital-temporal ventral, del hemisferio izquierdo.

Aplicación: la introducción de cada letra va acompañada de los comandos del profesor para que el niño la trace con el dedo, siguiendo la dirección de los rasgos (al mismo tiempo emite el sonido que concretiza el fonema representado por el grafema). La información sensorial procesada por la visión, el oído, el tacto, la cinestesia y la propiocepción se refuerzan mutuamente, de acuerdo con los preceptos de Montessori.

- El sistema escrito del portugués, al igual que el español, es alfabético del que se origina otra gran dificultad para que un niño se vuelva alfabetizado, ya que percibe el habla como un continuo, es decir, no 
hay separación entre palabras, ni contraste entre consonantes y vocales. Por ejemplo, ¿por qué el niño, al comenzar a escribir, coloca una sucesión de signos en una línea, sin espacios en blanco entre las palabras? ¿Por qué luego escribirá “zojos” y no "los ojos”? Porque así es como el percibe el habla.

Como el sistema es alfabético y no silábico, el niño tendrá que aprender a desmembrar la sílaba y reconocer el fonema: este aprendizaje, que se llama conciencia fonémica, sólo es posible, en un contexto lúdico, asociando cada grafema (concretizado por una o dos letras) a un fonema. ATENCIÓN: trabajar solo con sonidos aislados, o con los nombres de las letras, no es suficiente para preparar al niño para la alfabetización.

Un grafema, concretizado por una o dos letras, tiene el valor de un fonema, concretizado por un sonido; a veces, un grafema puede tener siempre el mismo valor, como $\langle\mathrm{f}\rangle$, pero otras veces puede tener más de un valor, como $\langle c\rangle$, que antes de los grafemas que representan las vocales posteriores y la semivocal, como /u/, /o/, /a/, /w/, tiene el valor de /k/, como en 〈cubo>, <costumbre>, <casa>, <cuica> y antes de los grafemas que representan las vocales y semivocales anteriores, como, por ejemplo, /i/, /e/, /j/, tiene el valor de /s/, como en 〈cine〉, 〈cera〉, 〈cielo>.

Veamos cómo se aplican estos principios en la primera Unidad del SSA: 


\section{Cuadro 1. Unidad 1 - VIVI}

Cuadro 1. Letras, palabras clave y números que aparecen en el Cuaderno de Ejercicios: Módulo 1, Lectura, del Sistema Scliar de Alfabetización, para que los niños tracen con el dedo, al mismo tiempo que emiten el sonido que concretiza el fonema, representado por el respectivo grafema, después de lo cual leen las palabras, aplaudiendo más fuerte, en la sílaba más intensa y más débil en las demás.
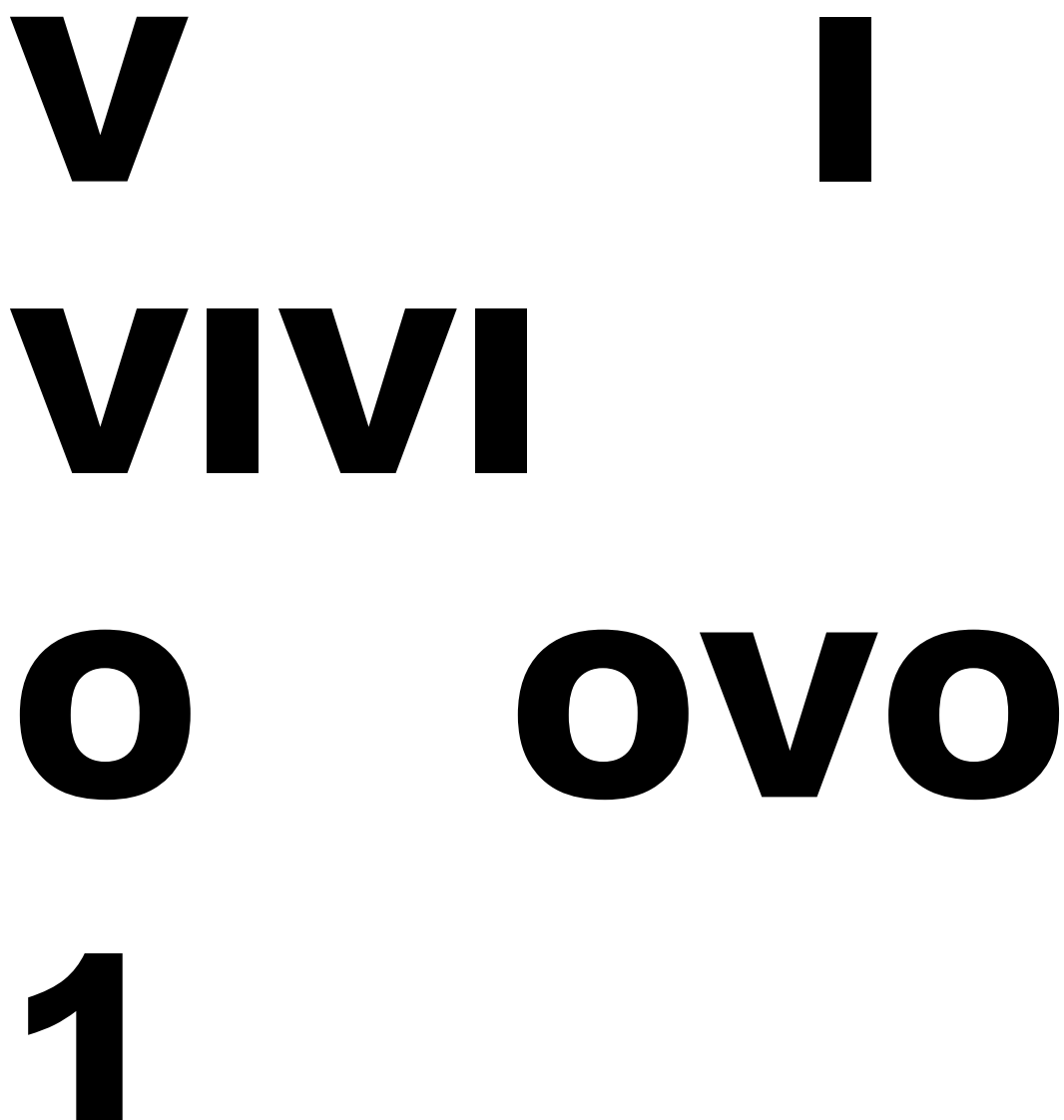

Fuente: Scliar-Cabral, 2020b, p. 6.

Luego, el maestro guía a los niños:

Conozcamos las letras y los sonidos que representan para poder leer. Abran la primera página: Con el dedo índice vamos a trazar la letra desde arriba hacia abajo y, después, subir desde abajo hacia arriba (señale la $\mathrm{V}$ en la pizarra, sin decir el nombre de la letra) y, al mismo tiempo, diciendo el sonido [vvv]. ¡Todo el mundo! Muy bien. Ahora, la letra en el costado (señale I en la pizarra), con el dedo índice vamos a trazar la letra desde arriba hacia abajo, diciendo [iii]. Ahora tracemos las dos letras (señale VI en la pizarra), diciendo [vi vi vi] (Scliar-Cabral, 2018, p. 11).

Ahora pasemos a la segunda sílaba (señale la segunda V de VIVI en la pizarra, sin decir el nombre de la letra). Con el dedo índice vamos a trazar la letra desde arriba hacia abajo y, después, subir desde abajo hacia arriba (señale la $\mathrm{V}$ en la pizarra, sin decir el nombre de la letra) y, al mismo tiempo, diciendo el sonido [vvv]. ¡Todo el mundo! Muy bien. Ahora, la letra en el costado" (señale I en la pizarra), con el dedo índice vamos a trazar la letra desde arriba hacia abajo, diciendo [iii]. Ahora tracemos las dos letras (señale VI en la pizarra), diciendo [vi vi vi] (Scliar-Cabral, 2018, pág.11). 
- Para reconocer la palabra escrita, separada por espacios en blanco, además de saber asignar los valores a cada grafema, el niño debe saber dónde cae el acento más fuerte (acento de intensidad), porque en portugués, como en español, el acento puede caer en la última (oxítona), penúltima (paroxítona), o antepenúltima sílaba (proparoxítona).

Este aprendizaje requiere el desarrollo de la conciencia fonológica. Vea cómo se aplica en el SSA. Luego, el maestro guía a los estudiantes que usarán la hoja del Libro de trabajo, como se muestra en la Tabla 1:

"¡Listo! Podemos leer muy rápidamente el nombre de la niña de nuestra historia” (guíe a los alumnos a no detenerse en cada sílaba): "VIVI. Ahora, leamos el nombre muy rápido, aplaudiendo más suave en la primera parte y más fuerte en la última parte". (Scliar-Cabral, 2018, pág.11).

En conclusión, la estrategia de enseñanza-aprendizaje se basa en un trípode de conceptos solidarios:

1 - Reconocer cuales son, cuántos hay y cómo se combinan los rasgos invariantes de las letras del alfabeto latino, centrándose en la dirección de tales s que las diferencian;

2 - Reconocer los grafemas, asociándolos con los fonemas que representan: tanto los fonemas cuanto los grafemas sirven para distinguir significados, por lo tanto, las letras que concretizan estos últimos deben estar dentro de palabras y estas en un texto;

3 - Para reconocer la palabra escrita, es necesario saber asignar dónde cae el acento de mayor intensidad.

Los niños tendrán a su disposición un libro de cuentos, Aventuras de Vivi (Scliar-Cabral, 2020a), con muchas ilustraciones, con las palabras que ya saben leer en negrita: luego se hace una lectura interactiva con el maestro, quien les da el siguiente comando:

Conozcamos la historia de una chica llamada ... Cuando las letras son más negras, las lees todas juntas. Me detengo para que lean: Empecemos por el título, todos: VIVI

Érase una vez una chica llamada VIVI. (Scliar-Cabral, 2018, p.12).

- El habla presenta variedades determinadas por varios factores: al leer, el niño convierte lo que lee a su variedad sociolingüística (respetándolo al leer en voz alta); cuando escribe ocurre lo contrario: el sistema escrito es uno en todo el territorio de habla portuguesa.

Aplicación: debido a la movilidad social, hay estudiantes que provienen de diferentes regiones e incluso de familias que practican otros idiomas en casa, como italiano, alemán, polaco o japonés; también existen diferencias determinadas por el nivel de educación y educación de los padres. Por eso, en la escuela se debe enseñar el respeto a las diferencias, evitando que estos niños sean ridiculizados cuando hablan o leen en voz alta. 
- La alfabetización integral se basa en el supuesto de que el objetivo es la educación integral del individuo: la cognición, el afecto, la sociabilidad, lo físico y lo estético, en vasos comunicantes, deben conducirlo al ejercicio de la ciudadanía y la realización personal, con la capacidad de comprender los textos escritos que circulan en la sociedad y de producir los de que necesite. Esto significa que las ciencias humanas no pueden separarse de las ciencias biológicas: no se puede pensar lo cultural sin la especialización biológica, ni cerebral sin dejarse moldear por el entorno.

En todas las Unidades se realizan actividades de música, danza y artes plásticas, como en la Unidad 1, en la que los niños, en torno a la canción "Marcha soldado", practicaron con la banda interpretada por los niños y precedida de la actividad de los niños confeccionando sombreros (resaltar la figura del triángulo) con hojas de periódico; pueden, además, improvisar una coreografía. El maestro, al frente, debe marcar el ritmo, tocando el instrumento triangular.

La alfabetización integrada es aquella que aprovecha todos los espacios y tiempos disponibles para la enseñanza-aprendizaje de la dirección de los rasgos que diferencian las letras entre sí, la constitución de estas en grafemas asociados a sus respectivos valores, los fonemas, ambos con la función de distinguir significados, por lo tanto, insertados en palabras y estos en textos que son significativos para el alumno, es decir, utiliza las asignaturas de matemáticas, ciencias, estudios sociales, educación física, artes, ocio y actividades de socialización, todo ello enredado coherentemente en torno a un tema, con un objetivo común. La integración entre escuela, familia y comunidad es fundamental para el éxito de la propuesta. Los aspectos ecológicos no se han olvidado.

\section{EXPERIMENTOS EM EL NORESTE BRASILEÑO}

Con base en los resultados de la Evaluación Nacional de Alfabetización 2016 (INEP, 2018), que evaluó a más de dos millones de niños de primaria (EF) al final del 3er año, en el que Sergipe (SE) ocupó el último lugar en lectura (3,02\%). y Alagoas (AL), el peor, en escritura, con solo $1.68 \%$ y Sergipe el antepenúltimo lugar, con solo $1.84 \%$, en los niveles más altos de competencia), decidí priorizar la aplicación del SSA en estos dos estados del noreste (de que presentaré una cuenta más detallada en este artículo): las dos Secretarías Municipales de Educación que inicialmente aceptaron la propuesta fueron las de Lagarto, SE y São José da Laje, AL.

\section{METODOLOGÍA}

La investigación intervencionista se inició en 2017 en São José da Laje y Lagarto, con el aval del ejecutivo municipal, legislativo y judicial, con el consentimiento y adhesión de familiares y bajo la égida de las respectivas Secretarías Municipales de Educación (SEMED). Informaremos los instrumentos 
utilizados desde 2017 hasta finales de 2019. Los dividimos en aquellos para la formación de educadores y alfabetizadores, con la educación de los niños y en los que se utilizan para evaluar los resultados.

Para reconfigurar el proceso de alfabetización, la SEMED en São José da Laje ha adoptado en la formación continua de los educadores, desde 2017, los libros del SSA para apoyarlos e implementarlos, así como el material para el estudiante (Scliar-Cabral, 2013, 2018, 2020a, b), en aquel momento, ofrecido por el autor, en versión digital. La Dirección del Departamento General de Educación promovió la formación, en reuniones quincenales de cuatro horas, incluida la escucha de Scliar-Cabral, vía Moodle de la Universidad Federal de Santa Catarina.

La SEMED de Lagarto seleccionó tres escuelas: Escuela Municipal Raimunda Reis y Escuela Multivariante Manoel de Paula y Escuela Rosa Venerine, cuyos tres alfabetizadores fueron capacitados por el académico José Humberto dos Santos Santana. Después de la capacitación, solo quedaron dos maestras, Jaqueline da Silva Nascimento, con dos clases en Raimunda Reis y Patrícia Vieira Barbosa Faria, con una clase en Manoel de Paula. Cada una recibió las hojas de ruta del SSA para su aplicación en cada unidad.

A inicios del segundo semestre de 2017 se suspendió la beca académica de Santana y, como el municipio fue notando los avances, se garantizó la continuidad del proyecto, formalizado por ScliarCabral, bajo la coordinación de la Dra. Mariléia Reis, quien lo renombró como "Alfabetización con excelencia en Lagarto".

Scliar-Cabral, a través de Skype, comenzó a capacitar a las dos maestras Jaqueline y Patrícia ya las dos coordinadoras Maria da Piedade S. Oliveira y Luzineuma Matias dos Santos. A finales de 2017, los 70 alumnos pasaron al segundo año de primária, sabiendo leer con fluidez.

Scliar-Cabral y la Dra. Mariléia Reis de la Universidad Federal de Sergipe asistieron al cierre del año escolar en 2017 de las escuelas involucradas y, en una visita a la SEMED, se firmó un convenio para adoptar el SSA como política pública de alfabetización en Lagarto.

En 2018, la SEMED expandió el SSA a 34 clases, con 910 estudiantes de primer año. De estas, se seleccionaron 17 clases distribuidas en 12 escuelas, 3 en zona rural y 9 en la sede, atendiendo a 365 alumnos, con enfoque en alfabetización hacia la lectura.

El Más Alfabetización, PMALFA (CAED, 2018) garantizó un asistente en el aula y en la aplicación de evaluaciones diagnósticas de entrada, evaluación procesal y evaluaciones diagnósticas de salida, con una plataforma digital y una matriz de referencia que guiaron el trabajo de lectura y redacción del docente. La matriz de referencia de la Evaluación Nacional de Alfabetización (ANA), utilizada al final del ciclo de alfabetización (3 años), pasó a formar parte de la matriz por segundo año. 
El Censo Escolar del INEP es el principal instrumento de recolección de información sobre educación básica y la encuesta estadística educativa más importante de Brasil. Con base en los datos de la inscripción inicial, compara los datos sobre el movimiento de los estudiantes y el desempeño escolar al final del año escolar.

La Evaluación de fluidez CAED (2019) consistió en actividades individuales de fluidez de lectura, realizadas oralmente y registradas a través de una aplicación de smartphone, enviada para su análisis.

\section{RESULTADOS Y DISCUSIÓN}

Comenzaremos con el experimento en São José da Laje. Los niños dieron un salto gigante en la competencia lectora, considerando el nivel más alto y deseable alcanzado por solo el $1.39 \%$ de los estudiantes de 8 años en el tercer año, según la ANA de 2016: después de la adopción del SSA, el municipio saltó a $71 \%$ de alumnos, a los 7 años, en el mismo nivel, no en $3^{\circ}$, sino en el $2^{\circ}$ año de primaria.

El resultado en los primeros años de EF São José da Laje es fruto de un gran compromiso con la educación para la inclusión social, además de la inversión en formación docente semanal de ZOOM con la propia autora de SSA, impartiendo formación a distancia los martes de 7:00 pm a 8:30 pm, para los educadores de $2^{\circ}$ año y, los miércoles, para los educadores del $1^{\circ}$ año y trabajo de seguimiento continuo para monitorear todo el proceso de alfabetización por parte del Director de Educación de la SEMED, articulado con cada coordinador escolar: cada docente, basado en el conocimiento científico, ha colaborado para modificar el escenario de São José da Laje y, en consecuencia, el escenario de Alagoas, que, a su vez, altera el escenario del Noreste. La exitosa experiencia, si se amplía, podría ayudar a erradicar el analfabetismo en Brasil a corto plazo.

Pasamos a los resultados y discusión del experimento en Lagarto. Según el indicador de flujo del Censo Escolar (INEP, 2018), de los 905 matriculados se aprobaron 898 alumnos de 1er año de EF, con $99,2 \%$ del total y $0,0 \%$ reprobados y $0,8 \%$ ya no asistidos. El indicador de aprendizaje está relacionado con los resultados de las evaluaciones externas realizadas en las clases que completan alguna etapa de la docencia, tales como: $2^{\circ}$ año (cierra el ciclo de alfabetización). Las evaluaciones externas se consideran un termómetro de cómo va el proceso de aprendizaje. Así, es a partir de los resultados obtenidos por las evaluaciones externas que cada escuela, municipio, estado y país es capaz de analizar y luego crear estrategias para alcanzar las metas establecidas.

En la evaluación del desempeño en lectura de ANA de 2016, había cuatro niveles, siendo el más bajo el 1 (prácticamente analfabeto), en el estado de Sergipe con un porcentaje muy alto de 45,28\% y en el 4, el nivel de competencia deseable para un niño de 8 años, solo el 3.02\%, al finalizar el 3er año de la Escuela Primaria. En la comparación de tales resultados, con los obtenidos en la evaluación de tres niveles, 
en el Programa Más Alfabetización (CAED, 2018), ahora, al finalizar el $2^{\circ}$ año de primaria, con niños de 7 años, en los dos colegios de Lagarto, también en el estado de Sergipe, que han adoptado la SSA desde 2017, constatase que, en el nivel 1, el más bajo (prácticamente analfabetos) hubo un porcentaje de solo 8,7 y 9,1, mientras que en el nivel deseado, 3, el más alto, en Más Alfabetización, ¡las dos escuelas alcanzaron el 34,8 y el $31,8 \%$

Tabla 2. Niveles de lectura en dos escuelas de Lagarto (SE)

\begin{tabular}{|c|c|c|c|c|c|}
\hline ESCUELAS & LOCAL & $\begin{array}{c}\text { NIVEL } \\
\mathbf{1}\end{array}$ & $\begin{array}{c}\text { NIVEL } \\
2\end{array}$ & $\begin{array}{c}\text { NIVEL } \\
\mathbf{3}\end{array}$ & $\begin{array}{c}\text { NIVEL } \\
\mathbf{4}\end{array}$ \\
\hline \multicolumn{6}{|c|}{ ANA (2016) } \\
\hline Todas las escuelas avaliadas & Sergipe & 45.28 & 34.91 & 16.78 & 3.02 \\
\hline \multicolumn{6}{|c|}{ MÁS ALFABETIZACIÓN (2018) } \\
\hline Raimunda Reis & Lagarto & 8.7 & 56.5 & 34.8 & -- \\
\hline $\begin{array}{l}\text { Manoel de Paula Menezes } \\
\text { Lima }\end{array}$ & Lagarto & 9.1 & 59.1 & 31.8 & -- \\
\hline
\end{tabular}

Fuente: Inep (2018); Caed (2018).

En los resultados de la Evaluación de Fluidez (CAED, 2019), la Escuela Múltiple Adelina Maria de Santana Souza (EMAMSS), que se benefició del SSA, alcanzó el puntaje más alto en el nivel 3, fluidez 42.3\%, entre todas las escuelas municipales de Lagarto, en el segundo año, como se muestra en la Tabla 2.

\section{CONCLUSIONES}

El objetivo de este artículo fue resaltar los principios del Sistema Scliar de Alfabetización, creado para ayudar a erradicar el analfabetismo funcional en Brasil.

La investigación intervencionista se llevó a cabo en dos municipios del noreste de Brasil, São José da Laje, en el estado de Alagoas y Lagarto, en el estado de Sergipe, que consistió en la formación continua en línea semanal de educadores involucrados en la alfabetización de $1^{\circ}$ y $2^{\circ}$ año Primaria, en escuelas públicas, con metodología y material pedagógico innovadores, basados en avances en neurociencia, lingüística, psicolingüística y neurociencia. Los instrumentos de evaluación de resultados fueron la Evaluación Nacional de Alfabetización, el Más Alfabetización y la prueba de Fluidez, todos del Ministerio de Educación de Brasil.

Los resultados mostraron que los niños de São José da Laje dieron un salto gigante en la competencia lectora, considerando que el nivel más alto deseable solo lo logró el 1.39\% de los estudiantes de 8 años en el tercer año, según la ANA, 2016: después de la adopción de la SSA, el municipio saltó al 
$71 \%$ de los estudiantes, a los 7 años, en el mismo nivel, no en el $3^{\circ}$, sino en el $2^{\circ}$ año, según el Mais Alfabetización, en 2018.

Según la ANA, 2016, en el nivel 1, el más bajo, hubo un porcentaje de 45,28\% en Sergipe, que, según el Más Alfabetización, 2018, bajó a 8,7 y 9,1\% en las dos escuelas de Lagarto que adoptaron la SSA; en el nivel de competencia deseable para un niño de 8 años, solo había un 3,02\% al finalizar el 3er año de Primaria, en el estado de Sergipe, mientras que en el nivel deseado, 3, el más alto, por el Más Alfabetización, las dos escuelas alcanzaron el 34,8 y el 31,8\%.

Los resultados muestran que es posible erradicar el analfabetismo funcional, si se produce un cambio en las políticas públicas de alfabetización, a favor de la formación continua de alfabetizadores y de metodologías y material pedagógico, basados en las ciencias que abordan el lenguaje verbal. 


\section{REFERENCIAS BIBLIOGRÁFICAS}

Caed. (2018). Mais Alfabetização, 2018. Disponível em: <https: //mais alfabetizacao.caed digital.Net /\#!/resultado-rede>. Acesso em 10 de set. 2020.

Caed. (2019). Fluência 2019. Disponível em: <Fluência | Programa fluência caeddigital.net>. Acesso em 06 de jan. 2020.

Dehaene, S. (2012). Os neurônios da leitura - Como a ciência explica a nossa capacidade de ler. Consultoria, trad. e supervisão de Scliar-Cabral, L. Porto Alegre: Penso.

Inaf (2018). Inaf Brasil 2018 Resultados preliminares.São Paulo: Instituto Paulo Montenegro.

Inep. (2018). Relatório SAEB/ANA 2016: panorama do Brasil e dos estados. Brasília: Instituto Nacional de Estudos e Pesquisas Educacionais Anísio Teixeira.

Montessori, M. (1997). Basic ideas of Montessori's educational theory: extracts from Maria Montessori's writings and teachings. Comp. Paul Oswald; GÃ/4Inter Schlz-Benesch. Trans. Lawrence Salmon. Oxford: Clio Press.

Rodríguez, P. T. (2021). La búsqueda de un modelo de evaluación competencial en educación básica. Un estudio de caso en el colegio los pinos de Algeciras. South Florida Journal of Development, Miami, v. 2, n. 4, jul./sep., 5851-5869. DOI:

$10.46932 / \mathrm{sfjdv} 2 \mathrm{n}$.

Scliar-Cabral, L. (2003). Guia prático de alfabetização - baseado em Princípios do Sistema Alfabético do Português do Brasil. São Paulo: Contexto, 2003 b.

Scliar-Cabral, L. (2013). Sistema Scliar de Alfabetização - Fundamentos. Florianópolis: Editora Lili.

Scliar-Cabral, L. (2018). Sistema Scliar de Alfabetização - Roteiros para o professor: Módulo 1, Volume 1. Florianópolis: Editora Lili.

Scliar-Cabral, L. (2020a). Aventuras de Vivi. Ilustrações de Juliana Morozowski. Florianópolis: Editora Lili.

SCLIAR-CABRAL, L. (2020b). Sistema Scliar de Alfabetização - Caderno de Atividades: Módulo 1, Leitura. Florianópolis: Editora Lili. 